\title{
Current status of multimodel superensemble and operational NWP forecast of the Indian summer monsoon
}

\author{
Akhilesh Kumar Mishra* and T N Krishnamurti \\ Department of Meteorology, Florida State University, Tallahassee, Florida 32306, USA. \\ *e-mail: akhil@io.met.fsu.edu
}

In the last thirty years great strides have been made by large-scale operational numerical weather prediction models towards improving skills for the medium range time-scale of 7 days. This paper illustrates the use of these current forecasts towards the construction of a consensus multimodel forecast product called the superensemble. This procedure utilizes 120 of the recent-past forecasts from these models to arrive at the training phase statistics. These statistics are described by roughly $10^{7}$ weights. Use of these weights provides the possibility for real-time medium range forecasts with the superensemble. We show the recent status of this procedure towards real-time forecasts for the Asian summer monsoon. The member models of our suite include ECMWF, NCEP/EMC, JMA, NOGAPS (US Navy), BMRC, RPN (Canada) and an FSU global spectral forecast model. We show in this paper the skill scores for day 1 through day 6 of forecasts from standard variables such as winds, temperature, $500 \mathrm{hPa}$ geopotential height, sea level pressure and precipitation. In all cases we noted that the superensemble carries a higher skill compared to each of the member models and their ensemble mean. The skill matrices we use include the RMS errors, the anomaly correlations and equitable threat scores. For many of these forecasts the improvements of skill for the superensemble over the best model was found to be quite substantial. This real-time product is being provided to many interested research groups. The FSU multimodel superensemble, in realtime, stands out for providing the least errors among all of the operational large scale models.

\section{Introduction}

Some of the basic characterizations of weather and climate systems over the region of the Asian summer monsoon may be obtained from Rao (1976) and Krishnamurti (1979) among several authors. Our study focuses on daily weather predictability over the diverse geographical regions of Asian monsoon. Most operational weather centers routinely provide 5 to 6 -day NWP forecast over the global domain. It is thus possible to examine the current state of the art skills of real-time operational global models over this belt. As many as seven global models were available for the prediction of weather on real-time. Our study entails the construction of ensemble and superensemble forecasts following Krishnamurti et al (2000a). Skills of member models and those of the ensemble and superensemble are constructed at the end of days $1,2,3,4,5$, and 6 of the forecasts. Traditionally, monsoon forecasts have encountered many difficulties that stem from numerous issues such as lack of adequate upper air observation over the neighboring oceans, mesoscale nature of convection, proper resolution, radiative interactions, planetary boundary layer physics, mesoscale air-sea fluxes, and representation of orography. Uncertainties in any of these areas lead to large systematic errors; those are reduced to some extent by the construction of the multimodel superensemble. These syntheses

Keywords. Monsoon forecast; ensemble modeling for monsoon; inspiring monsoon forecast beyond operational skills. 
of multimodel forecasts provide us with a somewhat better product for the current state of the art. In this paper, we present some of these current improvements that were made possible.

We shall next present the relative performance of several of these models with respect to each other. This will describe the current state of the art. Figure 1 shows the skills computed by EMC's global climate and weather modeling branch for January 2006. Models considered in this comparison study are global weather forecasting center models such as NCEP (GFS); ECMWF (ECM); UKMet (UKM); Meteorological Service of Canada (MSC) and NOGAPS (NGP). In figure 1(a) and (b) the anomaly correlations for the $500 \mathrm{hPa}$ geopotential height forecasts (day 1 through 6 ) are illustrated for the northern and southern hemisphere respectively. The forecast from the European center (ECMWF) carries the highest skills for all of the 6 days. It should be noted that these forecast validations were carried out using the analysis by NCEP/EMC and hence this is to be viewed as a current state of operational forecast skills. These skills for January 2006 for the northern hemisphere were slightly larger than those for the southern hemisphere. Higher skills for the anomaly correlation $>0.9$ were carried by the GFS (the US model), UKMet office model and the ECMWF through day 4 of forecasts over the northern hemisphere. These are indeed very high skills that have been achieved in recent years. Southern hemispheric skills are greater than 0.9 through day 3 of the forecasts by these models. Figure 1 (c and d) illustrates the skills of forecast over the tropics for zonal and meridional winds at the $850 \mathrm{hPa}$ and $200 \mathrm{hPa}$ levels for these same models through day 3 of forecasts. Anomaly correlation skills at these two vertical levels are comparable in magnitudes. The skills of the ECMWF again standout compared to all other models. Figure 1 (e through h) describes RMS error for the geopotential heights at the $500 \mathrm{hPa}$ level for the northern hemisphere and southern hemisphere as well as zonal and meridional winds at the $850 \mathrm{hPa}$ and $200 \mathrm{hPa}$ levels. In all of these illustrations, the least RMS error is systematically provided by the ECMWF. These errors grow with the lengths of the forecast periods, between day 1 and day 3 of forecasts. Figure 1 was extracted from the web files of NCEP/EMC in order to illustrate a current state of the art. The purpose is to show that it is possible to provide a multimodel consensus forecast on real-time that provides additional skill beyond what is seen here.

\section{Superensemble methodology}

Predictions from an ensemble of slightly different initial conditions and/or various versions of models using a single base model are often carried out by the weather services. An ensemble mean is defined as the average of all models involved in the ensemble suite. Another type of ensemble mean is the bias-removed ensemble mean, where the bias of each model is removed prior to the execution of an ensemble average. In both of these cases all models are given an equal weight of $1 / N$, where $N$ denotes the total number of models. The superensemble approach is a recent contribution to the general area of weather and climate forecasting developed at FSU; this has been discussed in a series of publications, Krishnamurti et al (1999, 2000a, 2000b and 2001). This technique entails the partition of a time line into two parts. One part is a 'training' phase, where forecasts by a set of member models are compared to the observed or the analysis fields with the objective of developing statistics (i.e., weights $a_{i}$ ) on the least squares fit of the forecasts to the observations. The second part is the forecast phase where estimates for $a_{i}$ from the training phase are used to create the superensemble. The performance of the individual models is obtained in the training phase using multiple linear regressions against observed (analysis) fields. The outcome of this regression is the weights assigned to the individual models in the ensemble, which are then passed on to the forecast phase to construct the superensemble forecasts. The temporal model anomalies of the variables are regressed against the observed anomalies when formulating the superensemble forecasts, and the weights are multiplied to the corresponding model anomalies. The constructed forecast is:

$$
S=\bar{O}+\sum_{i=1}^{N} a_{i}\left(F_{i}-\overline{F_{i}}\right),
$$

where $\bar{O}$ is the observed climatology over the training period; $a_{i}$ is the weight for the $i^{\text {th }}$ member in the ensemble; and $F_{i}$ and $\overline{F_{i}}$ are the $i^{\text {th }}$ model's forecasts and the forecast mean (over the training period) respectively. $N$ is the number of member models. The weights $a_{i}$ 's are obtained by minimizing the error term $G$, where $G$ is expressed as:

$$
G=\sum_{t=1}^{N_{\text {train }}}\left(S_{t}^{\prime}-O_{t}^{\prime}\right)^{2} .
$$

Here $N_{\text {train }}$ is the number of time samples in the training phase, and $S_{t}{ }^{\prime}$ and $O_{t}{ }^{\prime}$ are the respective superensemble and observed field anomalies at training time ' $t$ '.

This exercise is performed at all model grid points. A fit performed for all model variables at all model grid points at all vertical levels typically yields close to $10^{7}$ regression weights. These spread 


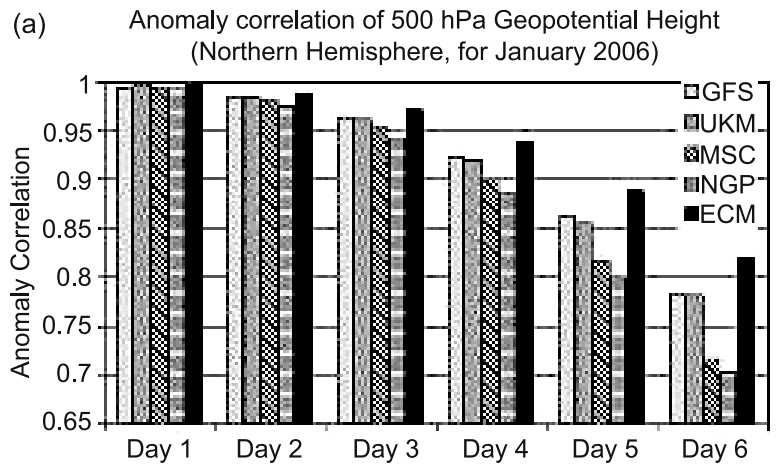

(b) Anomaly correlation of $500 \mathrm{hPa}$ Geopotential Height (Sourthern Hemisphere, for January 2006)

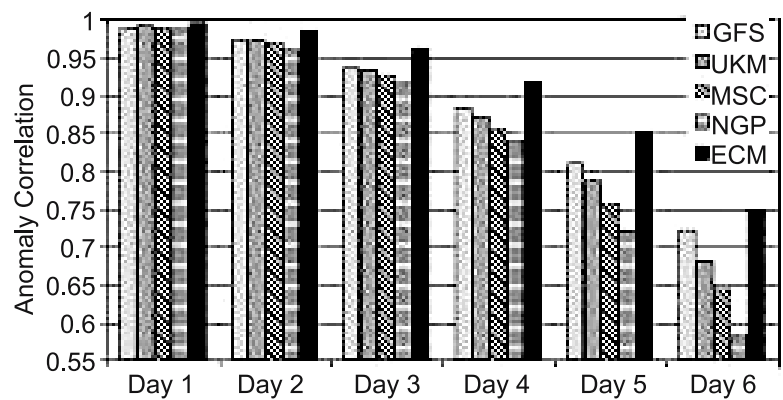

Anomaly correlation of $850 \mathrm{hPa}$

Zonal (left) and Meridional (right) Wind

(c) (Tropics only, for January 2006)

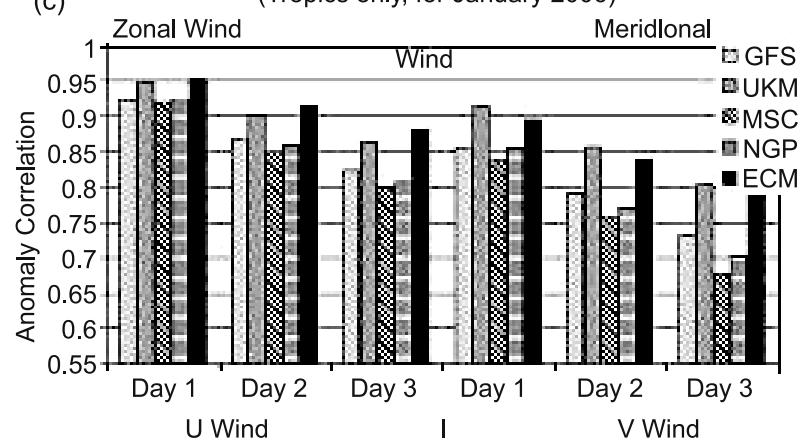
Zonal (left) and Meridional (right) Wind (Tropics only, for January 2006)

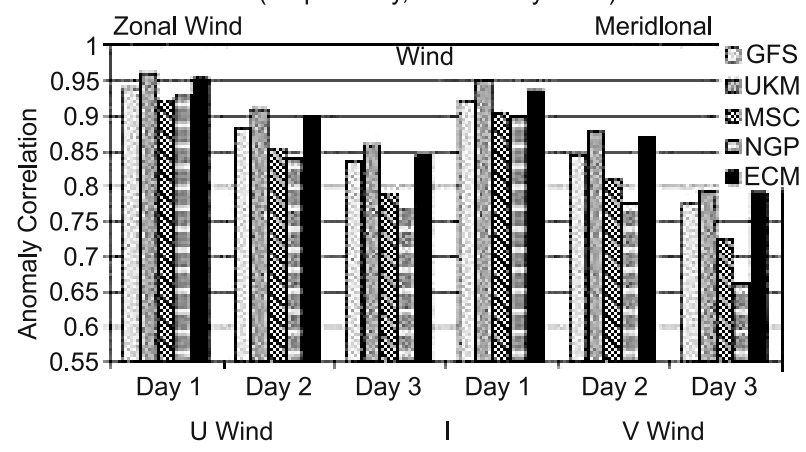

(e) RMS Errors of $500 \mathrm{hPa}$ Geopotential Height (Northern Hemisphere, for January 2006)

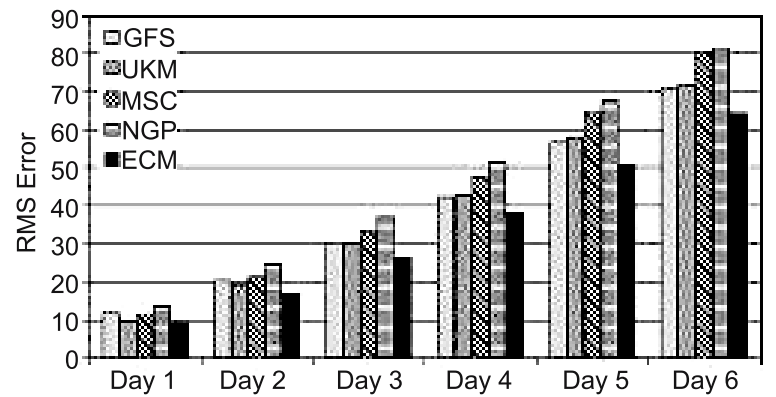

(f) RMS Errors of $500 \mathrm{hPa}$ Geopotential Height (Sourthern Hemisphere, for January 2006)

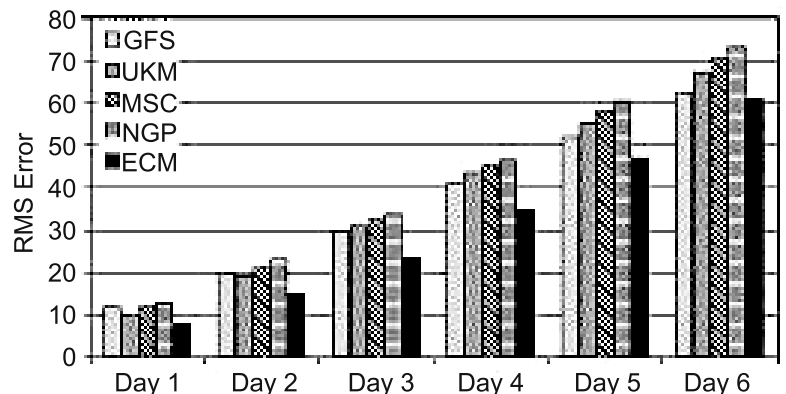

Root Mean Square Errors of $850 \mathrm{hPa}$ Zonal (left) and Meridional (right) Wind (Tropics only, for January 2006)

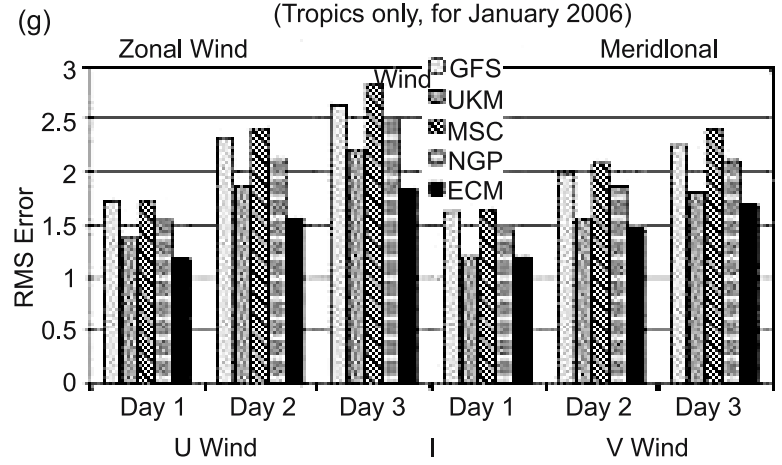
$\begin{array}{ll}\text { (h) } & \text { Root Mean Square Errors of } 200 \mathrm{hPa} \\ \text { Zonal (left) and Meridional (right) Wind }\end{array}$ (Tropics only, for January 2006)

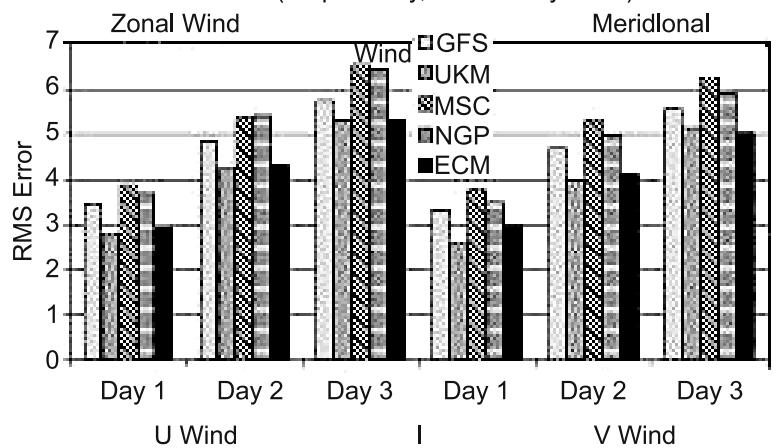

Figure 1. Current skills of the operational forecasting models for January 2006 (obtained from EMC/NCEP).

of weights are fractional, positive or negative. This large number arises from the number of transform grid points, number of vertical levels, number of basic variables and the number of models. Over many such locations we have noted diverse performance characteristics of the member models that 
arises from differences in horizontal and vertical discretization, treatment of physics, handling of inhomogeneity of land surface, orography, water bodies, surface physics and boundary conditions. All such peculiarities tend to leave their signature in the error distributions and hence on these weights. These may be thought of as a collective bias correction procedure. The second part of the time line is composed of model predictions. The superensemble approach combines each of these forecasts according to the weights determined during the training phase using the formulation. The prediction ' $S$ ' is referred to as the 'superensemble' forecast. This forecast should be contrasted with the more standard anomaly forecasts known as the bias-removed ensemble mean or ensemble mean forecast

$$
E=\bar{O}+\frac{1}{N} \sum_{i=1}^{N}\left(F_{i}-\bar{F}_{i}\right)
$$

or

$$
\hat{E}=\bar{O}+\frac{1}{N} \sum_{i=1}^{N}\left(F_{i}-\bar{O}\right) .
$$

The skill of the multimodel superensemble method significantly depends on the error covariance matrix, since the weights of each model are computed from a designed covariance matrix. The classical method for the construction of the superensemble utilizes a least square minimization principle within a multiple regression of model output against observed 'analysis' estimates. This entails a matrix inversion that is solved by the Gauss Jordan elimination technique. That matrix can be ill-conditioned and singular depending on the interrelationships of the member models of the superensemble. We have recently designed a singular value decomposition (SVD) method (Wilks 1995) for the multimodel superensemble that overcomes this problem and removes the ill conditioning of the covariance matrix entirely (Yun et al 2002). Tests of this method have shown great skills in weather and seasonal climate forecasts compared to the Gauss Jordan elimination method.

\section{Models participating in NWP superensemble suite}

Seven global models used in the construction of real-time superensemble include the European Center for Medium-Range Weather Forecasts model (ECMWF), the Global Forecast System of National Centers for Environmental Prediction (NCEP/GFS), Global spectral model of Japan Meteorological Agency (JMA), Global Environmental Multiscale Model from Canadian Meteorological Center (GEM), the Fleet Numerical
Meteorology and Oceanography Center model (NOGAPS), the model of Australian Bureau of Meteorology (GASP) and an FSU Global Spectral Model. In India, the National Center for Medium Range Weather Forecasting (NCMRWF) has been carrying out routine numerical weather predictions on real-time over the last 12 years. They issue medium range global forecasts, i.e., 1 to 6 days into the future. They have steadily improved the state of numerical prediction and its applications for the use of the agricultural community in India. The current FSU multimodel superensemble does not include this model within its suite of models. Thus a direct comparison of this model with the superensemble was not possible.

Table 1 illustrates the layout of the various participating models of our NWP Superensemble suite. This table shows the horizontal and vertical resolutions, various physical parameterization schemes and the land surface schemes deployed by each of these participating models. Overall, we note a diversity of operational models that carry a range of resolution and physics. Table 1 describes the models which were used to prepare the forecast used in this study, some of the operational centers are presently using an advanced version of the model.

\subsection{Data assimilation and satellite datasets}

A variety of data assimilation schemes are used by different operational NWP centers around the world. The initial datasets used by these models are generally the same for the surfacebased observations. However, the satellite-based data content of the different models do vary. Furthermore, there are major differences in the way the satellite data are being assimilated by the different centers. Direct use of radiances is part of the satellite data assimilation. NCEP and FNMOC/NOGAPS utilize the satellite-based datasets in their 3-DVar data assimilation system. JMA had been using 3-DVar until February 2005 in their global analysis system, although 4-DVar was operational in their mesoscale analysis system. From March 2005 onwards JMA has implemented 4-DVar in the global operational forecast and analysis system. ECMWF carried out very comprehensive 4-DVar data assimilation. Canadian model has 4-DVar operational; recently on 31 October 2006 they made major changes in their operational Global Environmental Multiscale (GEM) model that includes increasing significantly horizontal and vertical resolutions, improving its physical parameterization, improving the condensation and precipitation package, introducing the more sophisticated ISBA (interactions soil-biosphereatmosphere) surface scheme and improving the 


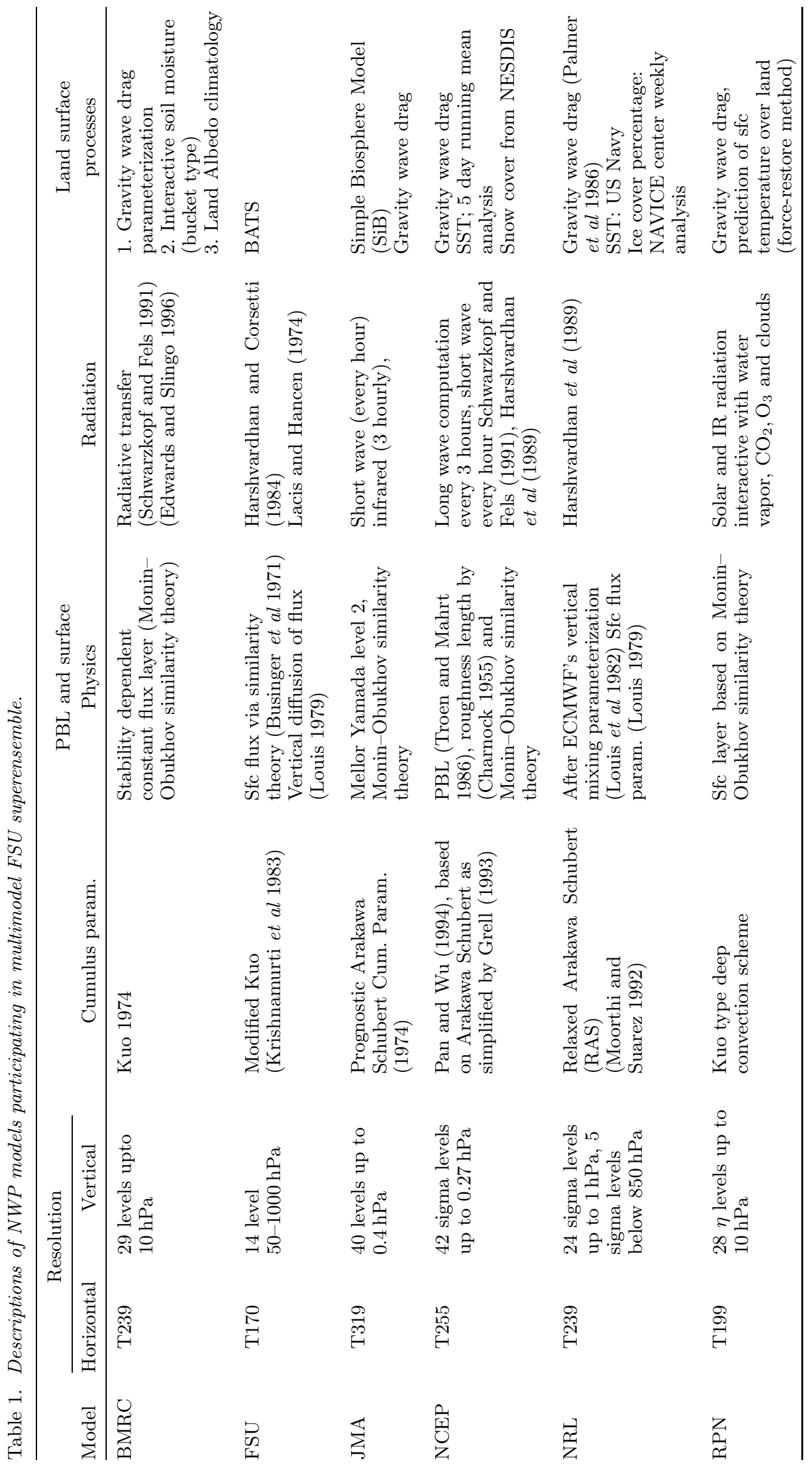


Table 2. ECMWF data assimilation system.

\begin{tabular}{|c|c|c|c|}
\hline \multirow[b]{2}{*}{ Conventional observations } & \multicolumn{2}{|c|}{ Satellite or instruments } & \multirow[b]{2}{*}{ List of sensors } \\
\hline & Platform & Data & \\
\hline $\begin{array}{l}\text { - Synop surface pressure } \\
\text { - Ship surface pressure and } \\
\text { wind } \\
\text { - Buoy surface pressure and } \\
\text { wind } \\
\text { - PAOB surface pressure } \\
\text { - Radiosonde temperature } \\
\text { wind and humidity } \\
\text { - Pilot wind } \\
\text { - Aircraft temperature and } \\
\text { wind American and European } \\
\text { profiler winds }\end{array}$ & $\begin{array}{l}\text { NASA- QuikScat } \\
\text { Geostationary platform } \\
\text { Polar platform }\end{array}$ & $\begin{array}{l}\text { Sea winds } \\
\text { AMVs + WV radiances } \\
\text { AMVs from MODIS- } \\
\text { TERRA, AQUA products }\end{array}$ & $\begin{array}{l}\text { - } 3 \times \text { AMSUA } \\
\text { - } 3 \text { SOAA } 15 / 16 / 17) \text { - radiances }(\text { F-13/14/15) - } \\
\text { radiances } \\
\text { - } 2 \times \text { HIRS } \\
\text { - } \text { ROAA-16/17) - radiances } \\
\text { (Met-7 GOES-10) } \\
\text { - } \text { Winds from } 3 \times \text { GEOS } \\
\text { (Met-7/5 GOES-10) and } \\
\text { - MODIS/TERRA } \\
\text { - Sea winds on QuikScat } \\
\text { - } \text { ERS-2 Altimeter/SAR } \\
\text { - } \text { SBUV (NOAA-16) } \\
\text { - } \text { AIRS AMSUA }\end{array}$ \\
\hline
\end{tabular}

GOME - Global Ozone Monitoring Experiment, SBUV - Solar Backscatter Ultraviolet, AMSUA - Advanced Microwave Sounding Unit-A, AIRS - Atmospheric InfraRed Sounder, HIRS - High Resolution Infrared Radiation Sounder, and ERS European Remote Sensing satellite.

model physics. In addition to the model changes, a number of improvements have also been introduced into the analysis used by the model, e.g., use of a new set of background error statistics. BMRC is using the 1-DVar in their operational Global Analysis and Prediction (GASP) system. BMRC is planning to adopt the 3-DVar, where a set of radiances is analyzed simultaneously in a three-dimensional sense. Table 2 describes the data assimilation system of ECMWF. Table 2 explains conventional observations (left column) and satellite data considered for the assimilation. Table 2 also explains list of sensors deployed in different platforms to facilitate the existing observing system.

\subsection{FSU model (physical initialization)}

The Florida State University Global Spectral Model (FSUGSM) also produces a 6-day forecast for inclusion into the superensemble. In this model, the initial fields have been physically initialized using the Ferraro and Marks rain rate algorithm (1995), also called the NOAANESDIS (National Oceanic and Atmospheric Administration)-(National Environmental Satellite Data and Information Service) SSM/I (Special Sensor Microwave Instrument) algorithm. Physical initialization is a powerful tool which primarily assimilates satellite-derived observed rainfall distributions along with calculated surface fluxes of moisture to produce a physically consistent and more realistic spin-up of the initial state. The key to the spin-up procedure is a Newtonian relaxation, where selected variables are 'nudged' toward prescribed values during a 24-hour pre-integration period. Upon integrating the FSUGSM, the rainfall patterns and surface moisture fluxes as well as the wind and mass fields appear more robust. For example, experiments have shown that within the ITCZ, monsoonal flows, and typhoons, the moisture flux is stronger and more organized. Thus, the precipitation areas are better defined. In the physical initialization technique, day minus one (one day prior to the initial condition) and day zero (initial time) ECMWF (European Centre for MediumRange Weather Forecasts) operational analyses are collected. Next, they are augmented with microwave radiance datasets from five satellites, including the NASA TRMM (Tropical Rainfall Measuring Mission) satellite. From these radiances, rain rates are derived. Then, via a number of reverse algorithms, including reverse similarity theory and reverse cumulus parameterization, the observed interpolated rainfall rates at each time step and at every location are used to derive a physically consistent initial state at hour zero. This initialization procedure includes the following five computational areas:

Step 1: The surface fluxes of moisture and heat are obtained from the vertical integration of the apparent moisture sink $Q_{2}$, and the apparent heat source $Q_{1}$, following the 
analysis of Yanai et al (1973). The proposed procedure using this moisture balance relation improves these estimates as one applies it repeatedly since the divergence, heating, and the moisture fields adjust to the imposed rain rates with time. The vertical integrals of $Q_{1}$ and $Q_{2}$ are carried out from the top of the atmosphere, $\sigma=0$ to the earth's surface $\sigma=1$, where $\sigma$ is the vertical coordinate.

Step 2: A reverse similarity theory is invoked during each time step of the physical initialization. The rain rates are assumed to be known and are used to provide the specific humidity and the potential temperature at the top of the constant flux layer. An assimilation of this data at the top of the constant flux layer promotes a consistency between the calculated flux and the imposed rain rate.

Step 3: A reverse cumulus parameterization algorithm restructures the moisture variable following the vertical coordinate which is consistent with the imposed rain rate. This restructuring of the moisture variable is achieved between the cloud base (the lifting condensation level) and the cloud top (the level at which the local moist adiabatic and the cloud-environment sounding intersect).

Step 4: In the upper troposphere $(\sigma<0.5)$ the moisture variable is restructured to minimize the local difference between the model and the satellite inferred fields of outgoing long wave radiation. A bisection method determines a parameter which defines the structure of the moisture variable in the upper troposphere.

Step 5: These components of physical initialization are executed within data assimilation phases of the model initialization. During this assimilation of the surface fluxes, the outgoing longwave radiation, the divergence field, the diabatic heating and the surface pressure evolves in a consistent manner. The details of the above procedure are presented in the two papers Krishnamurti et al (1991, 1993).

Lacking a unified column model that integrates all of the physical process (i.e., the surface flux parameterization, the cumulus parameterization and the radiative transfer scheme), the current FSU model has separate computational algorithms for these components. The sequence of computations within the physical initialization modifies the moisture variable separately to satisfy each requirement, i.e., fluxes, rain rate and the earth radiation budget. The sequence of reverse algorithms is: reverse similarity followed by OLR matching and then the reverse cumulus parameterization. The time interpolated rain rates are an input to the reverse similarity and the reverse cumulus parameterization algorithms. The satellite based OLR enters the matching algorithm. A one time step, forward integration follows the execution of the reverse cumulus parameterization algorithm. Since the reverse cumulus and the normal cumulus are carefully designed to be almost completely reversible, the "observed" rain rate is reproduced by the model at the end of the time step. Thus, an accumulation over 24-hour of simulated assimilated rainfall appears realistic.

Currently missed by the physical initialization are instances where the model calls for no precipitation (i.e., no net moisture convergence or absence of conditional instability) whereas the satellite or raingauge observations imply rain. We impose saturation as an upper limit for the modification of the specific humidity. This constrains the extent to which rainfall can be recovered in these instances. Further work is ongoing in both of these above areas to overcome these difficulties towards further improvement of the now casting skill.

\subsection{Optimizing the number of training days}

Because of the nature of atmospheric variability over different regions, the minimum number of training days required for stabilizing the statistical weights varies from region to region. Krishnamurti et al (2003) illustrated the nature of this geographical dependence. They studied the distribution of the minimum number of days that were required for the training phase of NWP multimodels. This distribution varies from roughly 90 days to 120 days; over the land area more days of training were required compared to those over the ocean. This led to our accepting a number of 120 days for the length of training the phase for NWP. We noted that roughly 120 days of training provided stability for the statistical weights. This number does vary somewhat from one vertical level to the next and from one atmospheric variable to the next. Overall a use of 120 days appeared to cover the needs for stable statistics. Another important aspect of these coefficients is that it is better to compute these from data of similar previous seasons. For instance a use of dry season pre-monsoon data over 120 days (such as March, April, May and June) may not be best suited for the rainy and wet months of July and August. In such instances, several previous years of July August datasets can be used. This of course also requires that the models of our forecast suite have not altered much over these several years used for the training phase. We have 
used the operational ECMWF analysis at $0.5^{\circ}$ latitude/longitude as the benchmark fields for most variables for the training phase. The observed measures of precipitation are derived from the so-called morphing technique of NOAA CPC $(\mathrm{CMORPH})$ that is described in some detail in Joyce et al (2004) and within the references stated therein. In the light of work by Krishnamurti et al (2000b, 2001), CMORPH dataset is used for training and validation of a new precipitation superensemble product in real-time.

\section{Results and discussions}

The anomaly correlation and the RMS errors for the sea level pressure, $500 \mathrm{hPa}$ level geopotential heights, and the zonal wind at the $850 \mathrm{hPa}$ level for the Asian monsoon domain covering the monsoon months June, July, August and September (2004) are illustrated in figure 2 (a through $\mathrm{f}$ ). The left panels carry the results for the anomaly correlation, and the right panels carry the RMS error. Figure 3 (a through f) illustrates the anomaly correlation and RMS error for the different components of the wind fields (i.e., the meridional wind at $850 \mathrm{hPa}$ level, the zonal wind at the $200 \mathrm{hPa}$ level, and the meridional wind at the $200 \mathrm{hPa}$ level). We note again similar very high skills for the anomaly correlations, $>0.9$ for day 4 of forecast for all these variables. In all cases, the RMS errors are the least for the multimodel superensemble compared to those of the member models and the ensemble. Forecasts for all these elements show very high skills for the multimodel superensemble that is shown by the dark bars. These carry the highest anomaly correlation and the lowest RMS errors for each of the forecast days. Here the skills for seven of the best models are compared with those of the superensemble (far right) and for the ensemble means (shown next to the superensemble). The results of the superensemble appear clearly better than those of the ensemble mean. The most striking results are the large improvements in the anomaly correlation from the multimodel superensemble, values as high as 0.90 to 0.94 on day 4 of the forecast are worth noting. The implications of such scores are discussed below.

Figure 4(a-b) (over plots) shows a superposition of the observed and predicted sea level pressure and $500 \mathrm{hPa}$ geopotential heights for a day 4 of forecasts when the anomaly correlation was of the order of 0.92 . The isobars of the forecasts, line for line, seem to lie on top of the observed isobars. We have chosen anonymous dates to show the pattern matching. The entire season of forecasts for day 4 and day 5 carry high anomaly correlations (see figure $4 \mathrm{c}-\mathrm{d}$ ). Figure $4(\mathrm{c}-\mathrm{d})$ depicts time series of the anomaly correlation of day 4 and day 5 MSLP forecast using the multimodel superensemble along with member models. This shows very high skills of the daily values. The member models do not carry that degree of consistency in their forecasts through days 4 and 5 . These skills show a spread between 0.35 and 0.85 on different days whereas that of superensemble stays close to 0.9 or higher in most days.

Monsoon lows and depressions are large scale disturbances with scale $>1000 \mathrm{~km}$. These are well resolved at the resolution of the multimodel superensemble (whose horizontal resolution is of the order of $80 \mathrm{~km}$ ). The consistent skill of the superensemble provides a useful guidance for the mesoscale modelers. Figure 5 was checked during day 4 where one such disturbance propagated across the Indian subcontinent (dotted lines are analysis while solid lines are day 4 forecast of multimodel superensemble) that occurred during 11 to 13 June 2004. Checking the anomaly correlation for these dates from figure $4(\mathrm{c}-\mathrm{d})$, we noted that in most of these instances the day 4 and day 5 of forecast skills of the anomaly correlations was around 0.9 for the multimodel superensemble. This shows that it is possible to predict the isobaric distribution during disturbance passages with a very high degree of accuracy. Figure 5 was deliberately selected to show the superposition of isobars (forecast and observed) on day 4 of the forecast where a depression was present over northern India. This shows the usefulness of the superensemble product. A high skill forecast for up to day 5 may not meet the needs of agriculture where at least two weeks forecast is generally demanded. However, it is satisfying to see that we can provide a very useful guide to the mesoscale modelers through day 5 . This overall consistent improvement of day-to-day skill for the superensemble is the special feature of these real-time products.

In the context of the usefulness of the forecasts of monsoon, precipitation is the most important variable. Precipitation forecasts are generally evaluated using a probabilistic skill, which is called the Equitable Threat Scores (see appendix 1). Skill score is evaluated for rainfall thresholds greater than a preset value. A bias score is also generally evaluated for each of the thresholds of rainfall amounts. The normalized bias score (see appendix 1) is designed such that a value of 1.0 defines a good forecast. Overestimates/underestimates of rainfall amounts carry bias score $>1$ or $<1$. In figure $6(\mathrm{a}-\mathrm{d})$, we present these results of equitable threat scores and the bias scores for the season (June to September 2004). We show here the equitable threat scores in the range of thresholds 
(a)
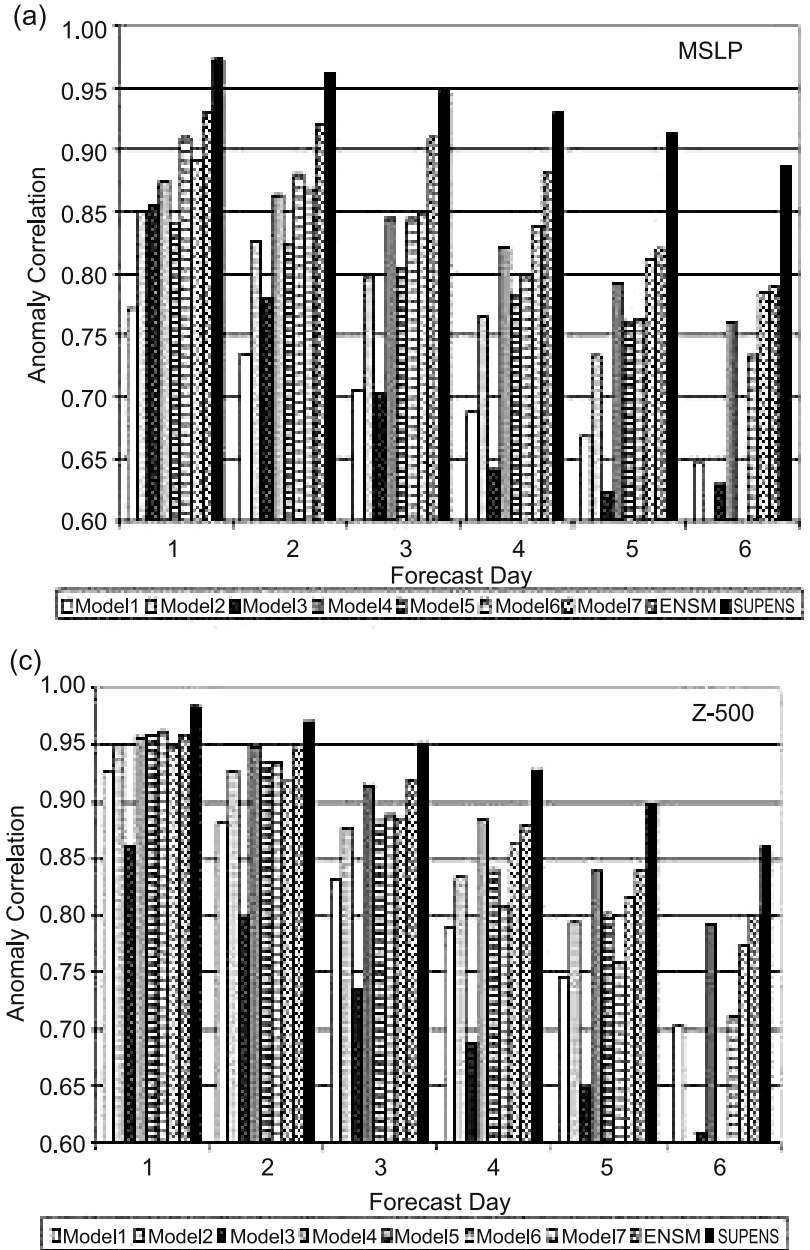

(e)

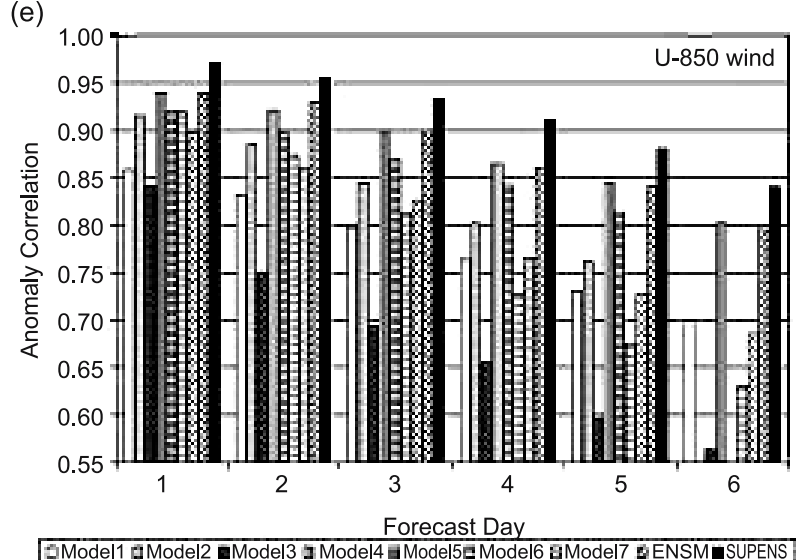

(b)

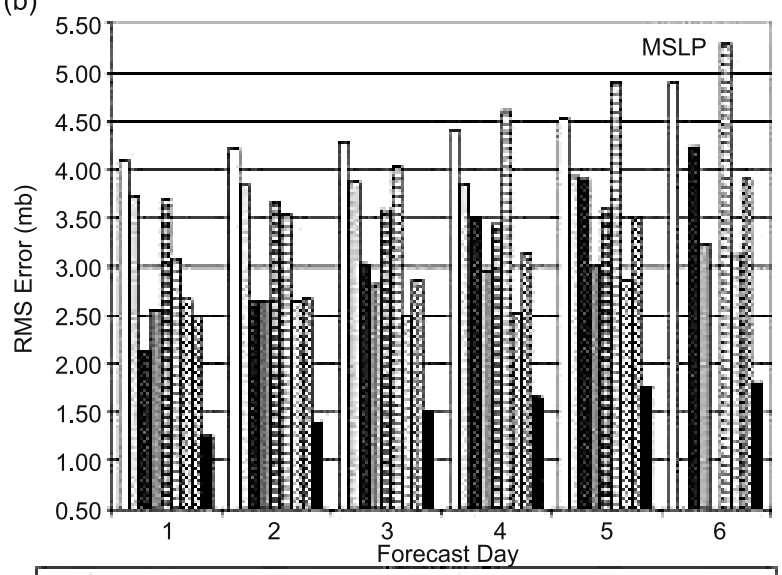

OModel 1 DModel 2 Model 3 \&Model 4 aModel 5 BModel 8 Model78ENSM SUPENS

(d)

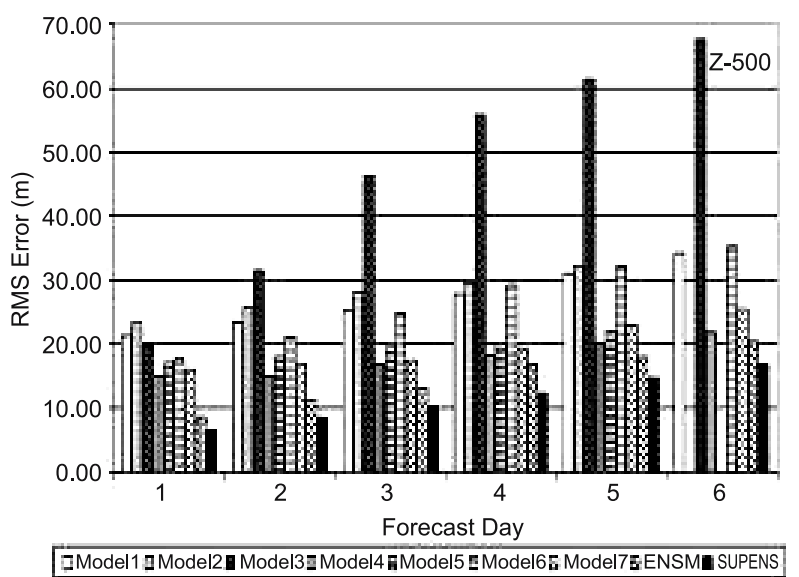

(f)

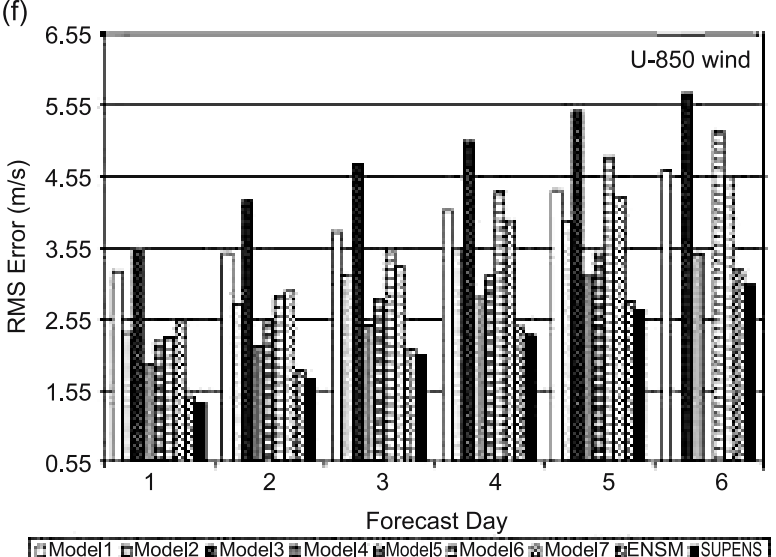

Figure 2. Forecast skills of the multimodel FSU superensemble compared with member models.

between 0.2 and $2.0 \mathrm{~mm} /$ day. The highest ETS are clearly carried by the multimodel superensemble compared to the individual models for day 1 through 5 of forecasts. The precipitation bias score of the multimodel superensemble is generally close to 1.0 for day 1 through 5 of forecasts; one of the member models does have a very high bias score, the performance of the bias score of the multimodel superensemble is close to that of the best model. A threshold of $0.2 \mathrm{~mm} /$ day implies the skill for the total rain whereas the thresholds $2 \mathrm{~mm} /$ day ignores the trace rain that many member models have an abundance of. The day 1 ETS for most member models ranges from 0.2 to 0.35 . With the multimodel superensemble, it is possible to retain a threat score greater than or equal to 0.3 through day 4 of forecasts. How good is an ETS of 0.3 ? That is the current now casting 
(a)

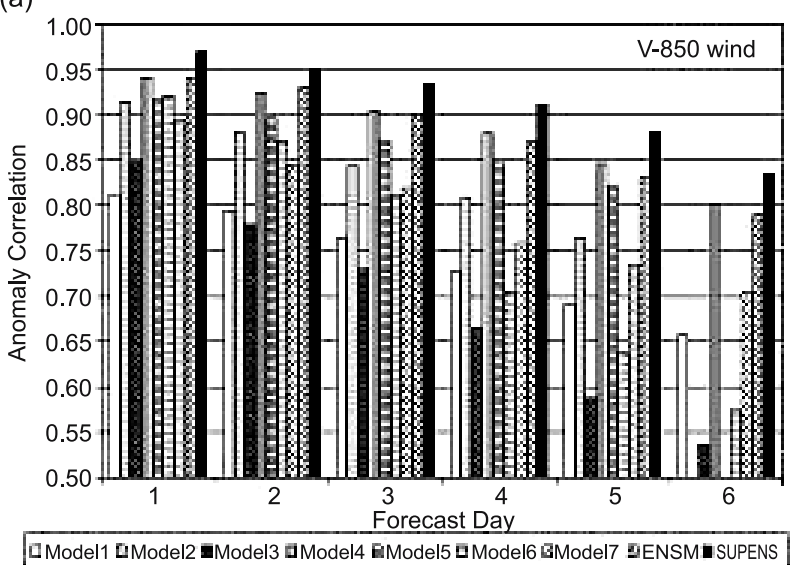

(c)

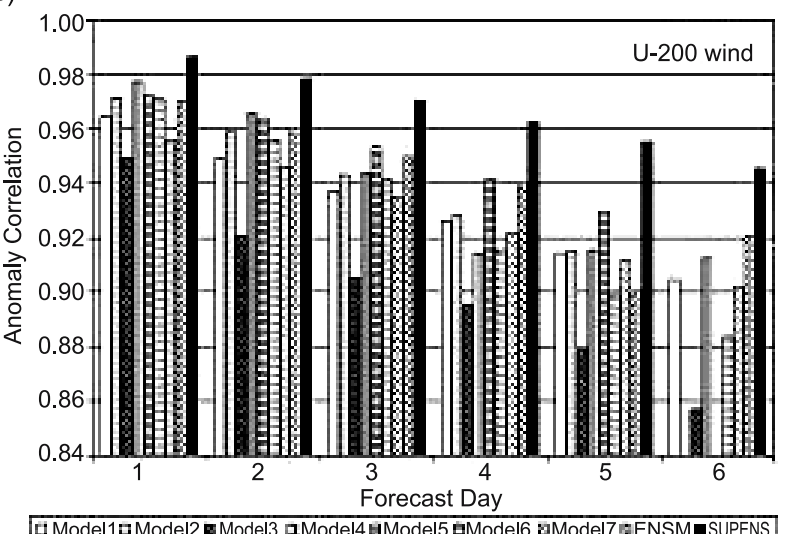

(e)

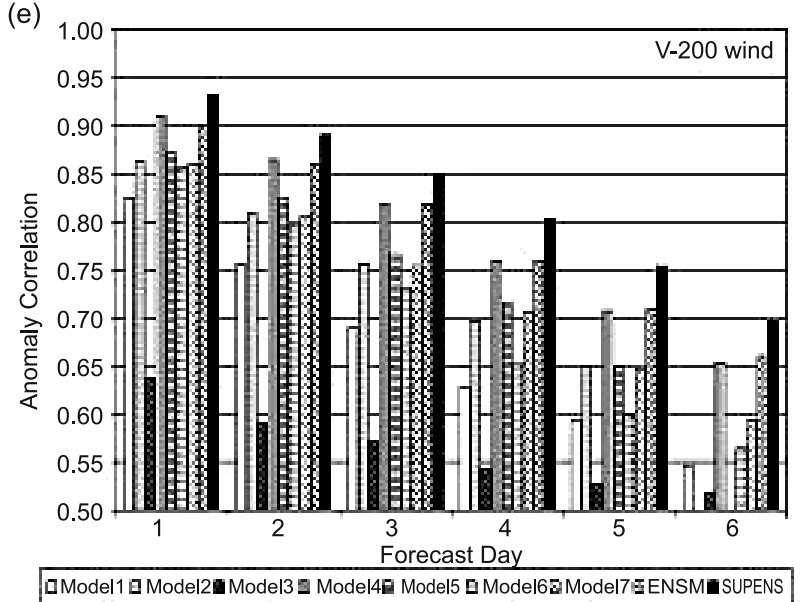

(b)

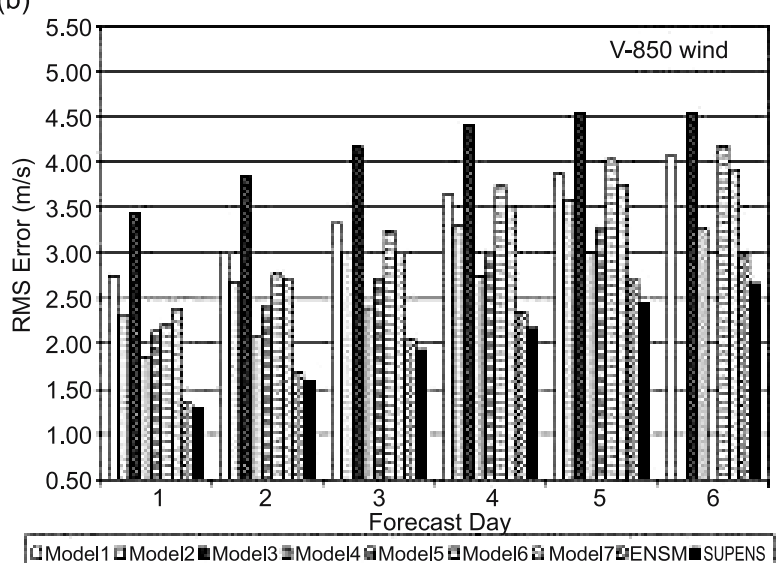

(d)

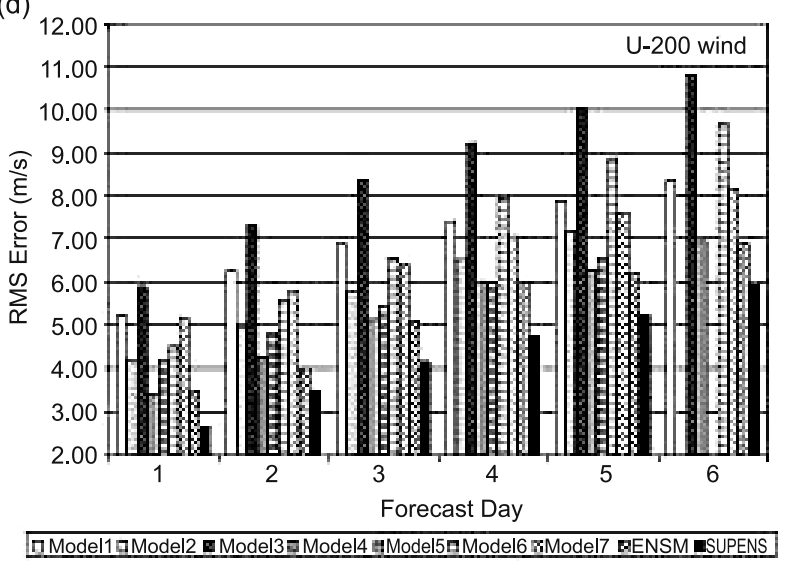

(f)

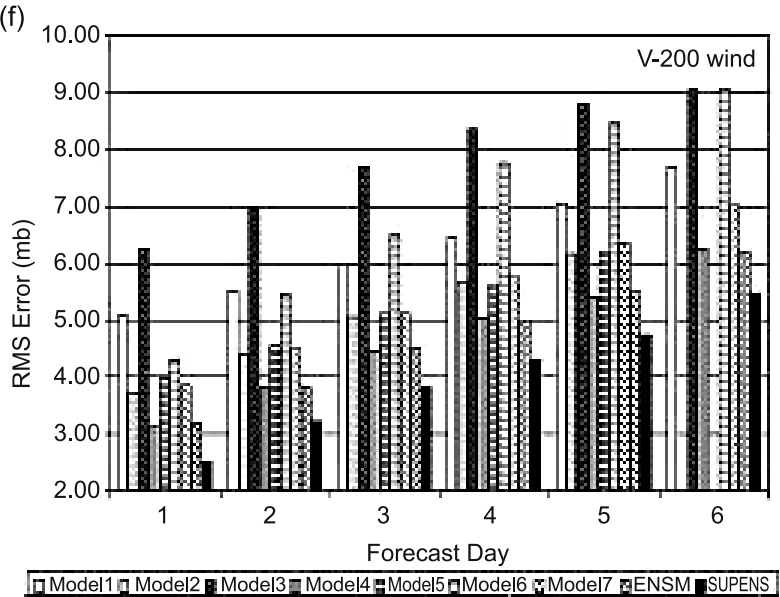

Figure 3. Forecast skills of the multimodel FSU superensemble compared with member models.

skill for most operational models. This score does signify some degree of usefulness of forecast over a large-scale model whose horizontal resolution is of the order of $100 \mathrm{~km}$. This skill cannot be directly compared to that of mesoscale models that are generally evaluated against radar-based rainfall estimates.

The ETS and bias scores for a case study of heavy rains from the passage of a monsoon depression are shown in figure $6(\mathrm{e}-\mathrm{h})$. Here the initial day
1 skills of the ETS for the multimodel superensemble for rainfall thresholds $>2.0$ and $>5.0 \mathrm{~mm} /$ day are both close to 0.4 . These skills are clearly higher than those for the member models. For this case study, there were noted minima of skill for day 3 of forecast. That sharp drop of skill between day 2 and day 3 of the member models was the period of very heavy rains where their bias scores (for days 2 and 3) showed values somewhat higher than 1.2. The bias score of the superensemble remained close 
(a)

Sea Level Pressure on

August $3^{\text {rd }} 2004 \mathrm{AC}=0.935$

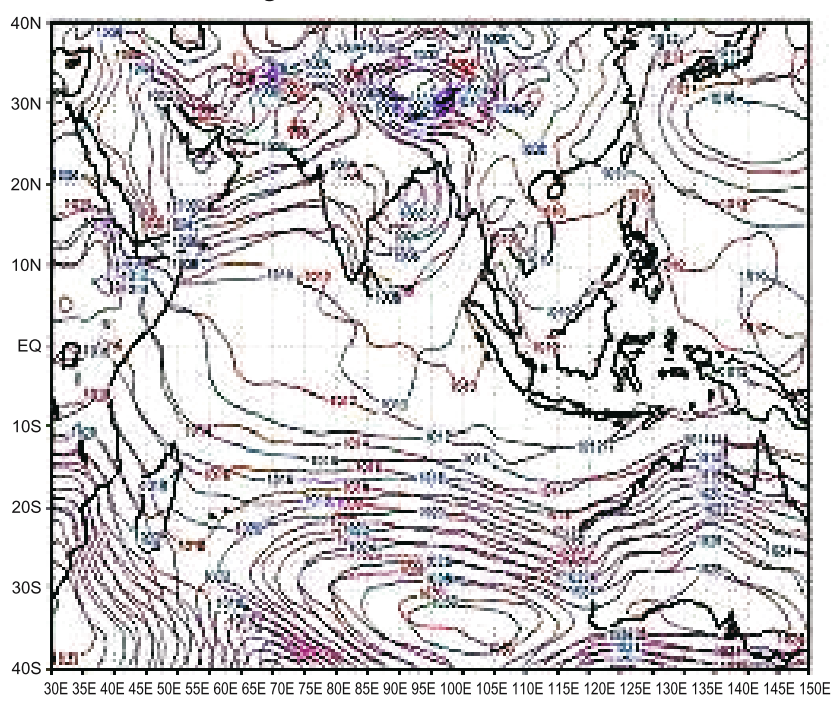

(b) $500 \mathrm{hPa}$ Geopotential heights on $25^{\text {th }}$ August 2004, AC $=0.924$

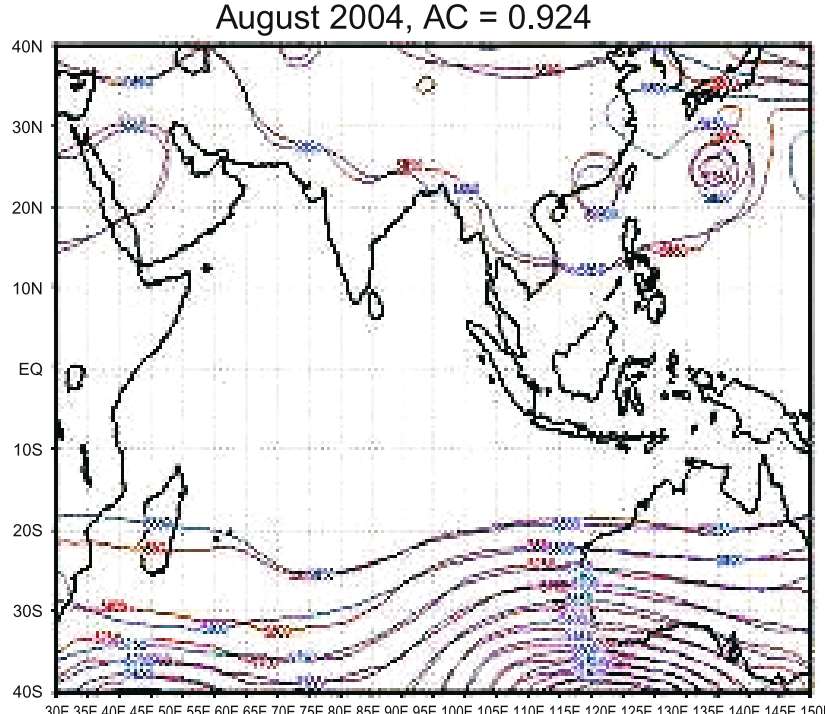

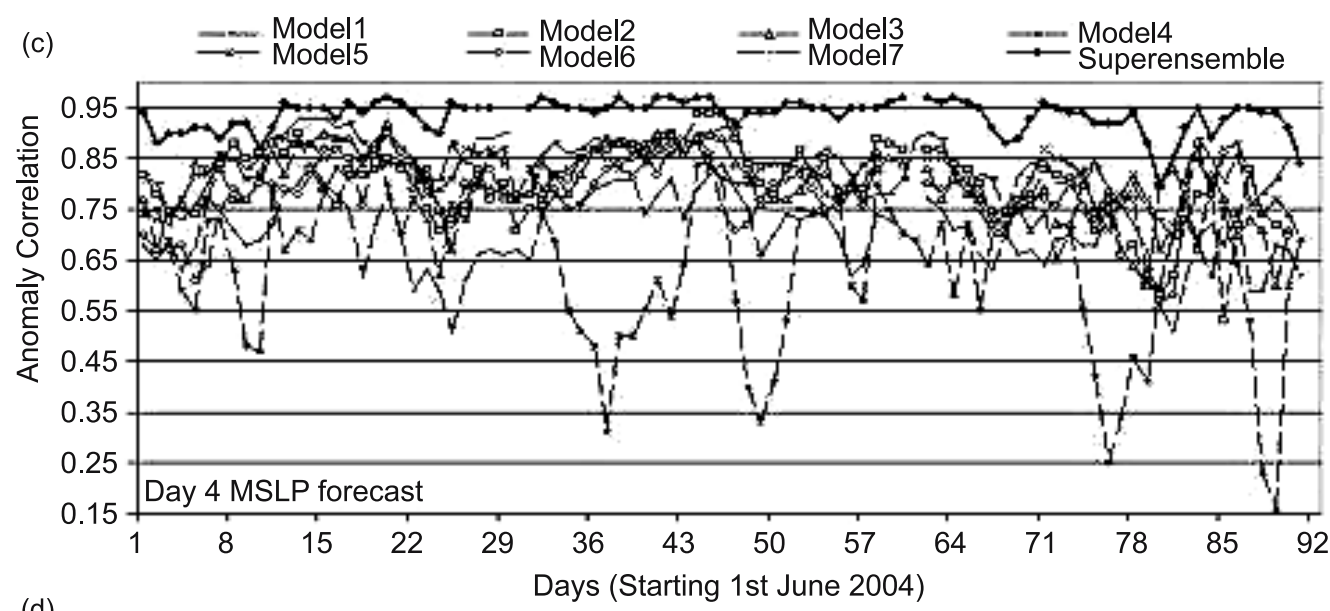

(d)

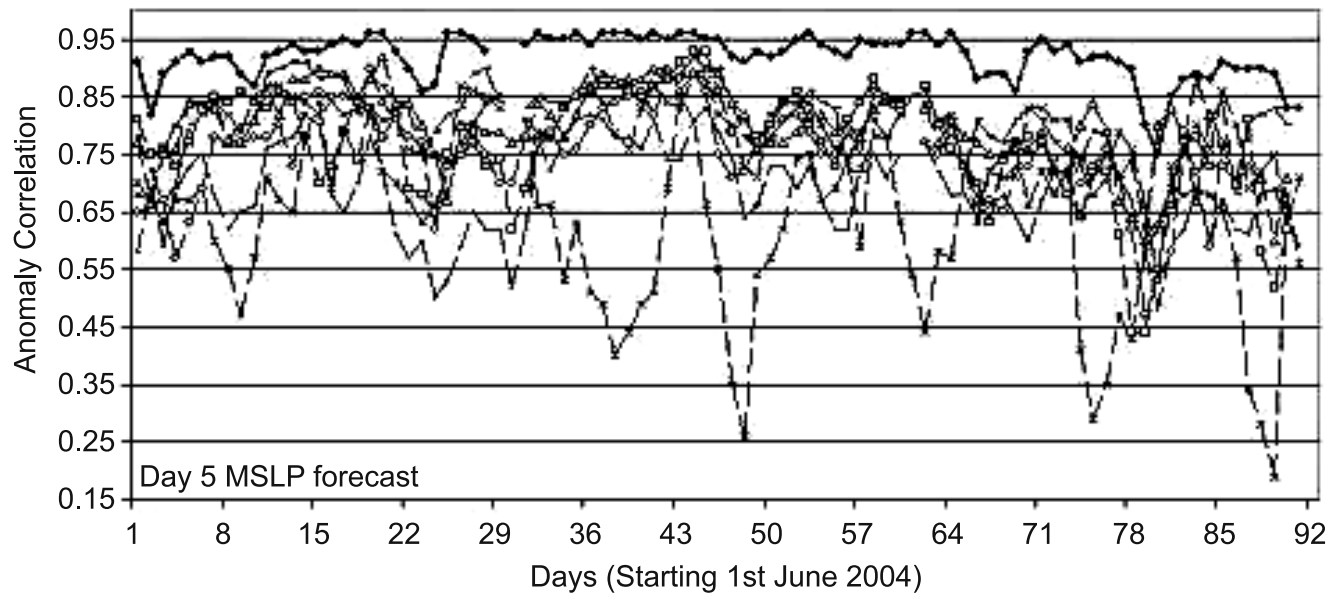

Figure 4. (a) Over plot of day 4 superensemble SLP forecast (red line) with analysis (blue line) on 3 August 2004, (b) Over plot of day 4 superensemble $500 \mathrm{hPa}$ geopotential heights forecast with analysis on 25 August 2004, (c) Day 4 MSLP anomaly correlation for 2004 monsoon season and (d) same as (c) except for day 5.

to 1.0 (for days 4 and 5 of forecasts). Overall, for this case study, the bias score of the multimodel superensemble were clearly better than those of the member models.

\section{Conclusion}

In medium range real-time global weather forecasts, the largest skill improvements are seen for 
(a)

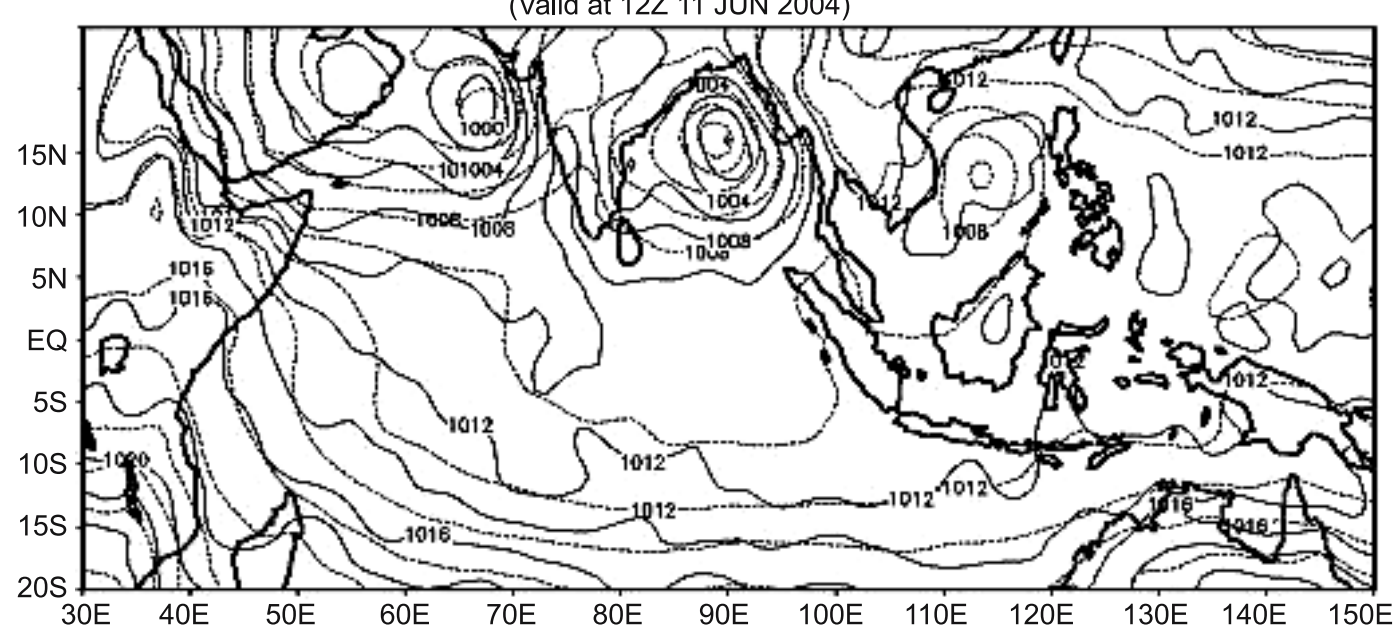

(b)

Analysis vs Day 4 Superensemble MSLP FCST

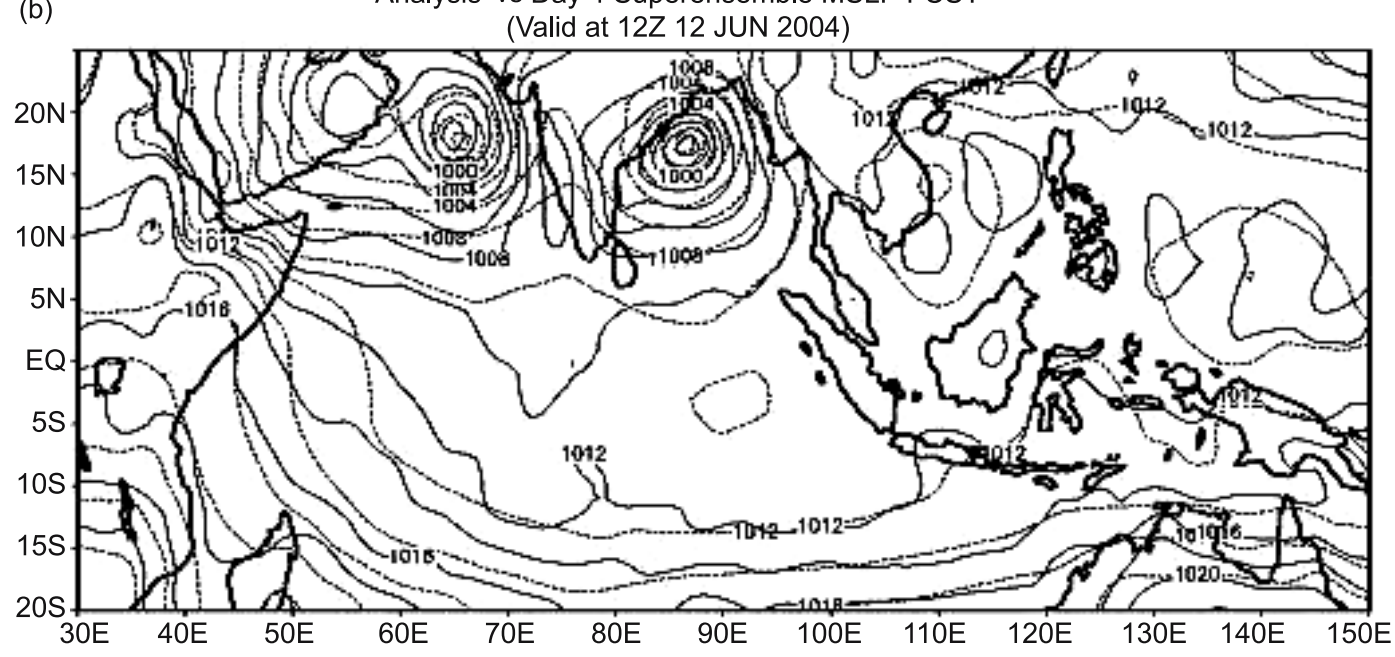

(c)

Analysis vs Day 4 Superensemble MSLP FCST

(Valid at $12 Z 13$ JUN 2004)

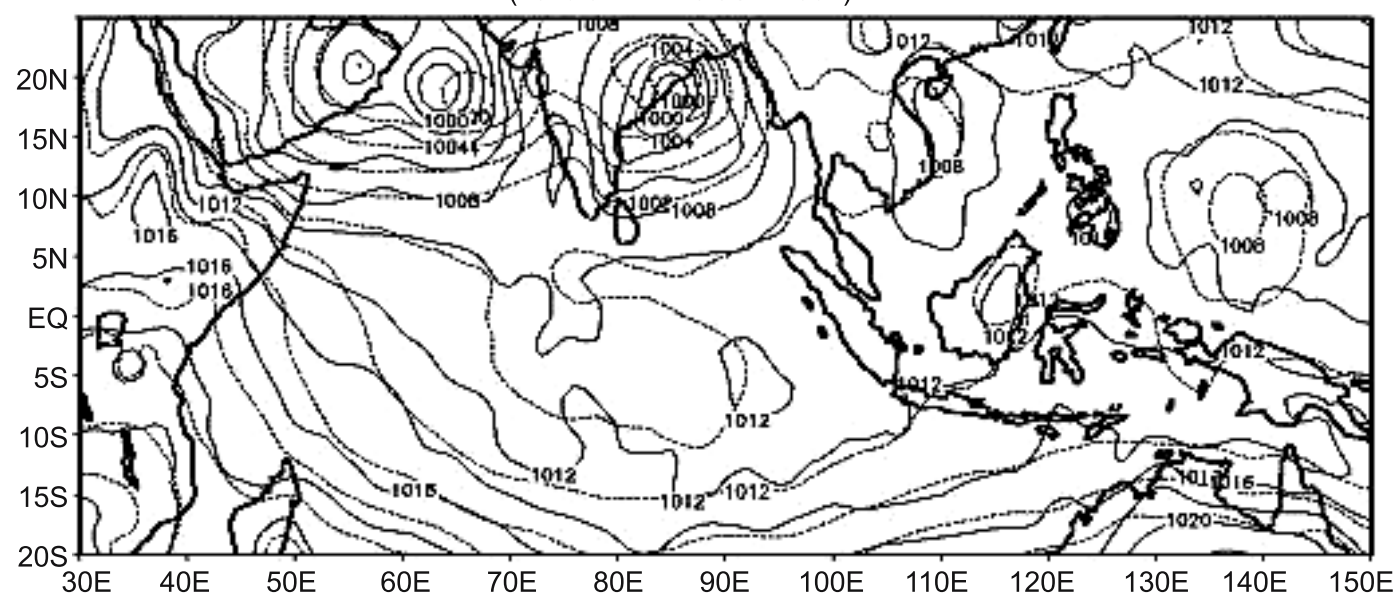

Figure 5. Analysis (dotted line) plotted against day 4 MSLP superensemble forecast (continuous line) during the passage of monsoon depression (a) 11 June, (b) 12 June and (c) 13 June 2004.

precipitation forecasts both regionally and globally. The overall skill of the superensemble is $40-$ $120 \%$ higher than the precipitation forecast skills of the best global models for precipitation forecasts.
The superensemble shows major improvements in skill for the divergent part of the wind and the temperature distributions. Tropical latitudes show major improvements in daily weather 
(a) Equitable threat score $(0.2 \mathrm{~mm} /$ day threshold $)$ June-September 2004 (30E-150E, 30S-40N)

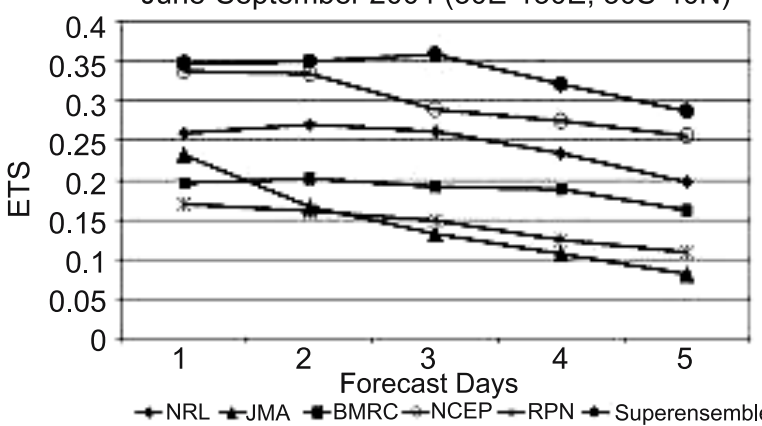

(c) Equitable threat score $(2.0 \mathrm{~mm} /$ day threshold $)$

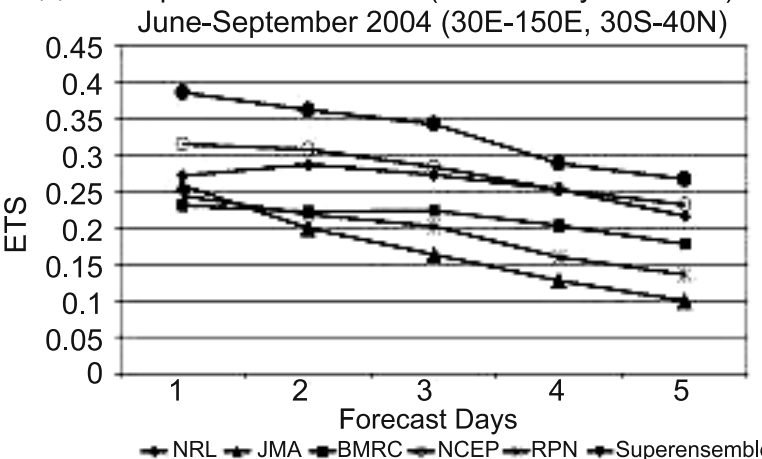

(e) Equitable threat score (2 $\mathrm{mm} /$ day threshold)

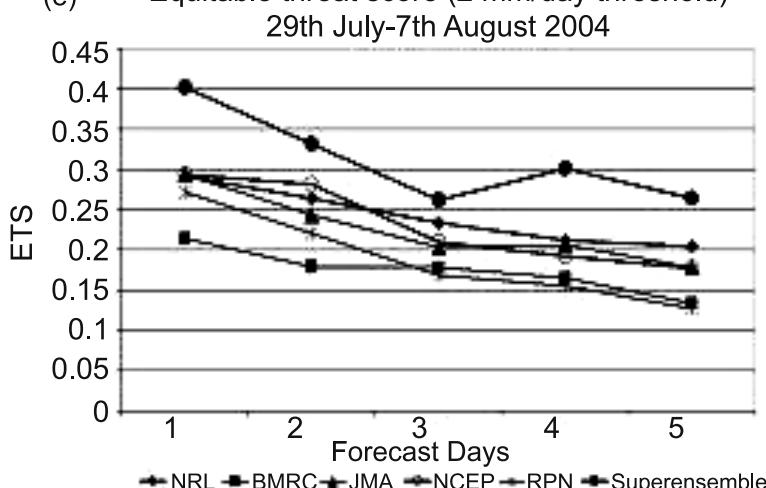

$\rightarrow \mathrm{NRL} \rightarrow \mathrm{BMRC}$ - JMA $\rightarrow \mathrm{NCEP} \rightarrow \mathrm{RPN}$ - Superensemble

(g) Equitable threat score $(0.5 \mathrm{~mm} /$ day threshold $)$ 29th July-7th August 2004

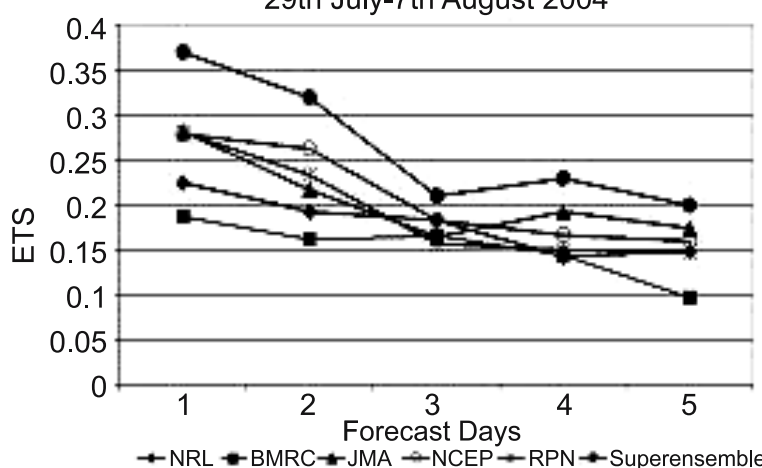

(b) Precipitation Bias score $(0.2 \mathrm{~mm} /$ day threshold $)$

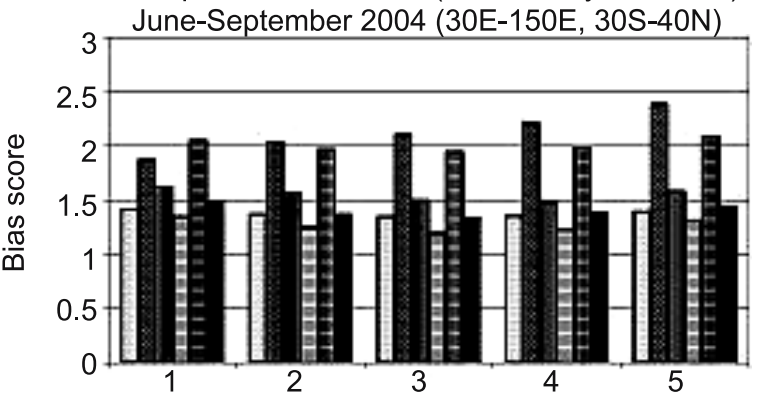

Forecast Days

a NRL a JMA aBMRC DNCEP aRPN — Superensemble

(d) Precipitation Bias score $(2.0 \mathrm{~mm} /$ day threshold $)$ June-September 2004 (30E-150E, 30S-40N)

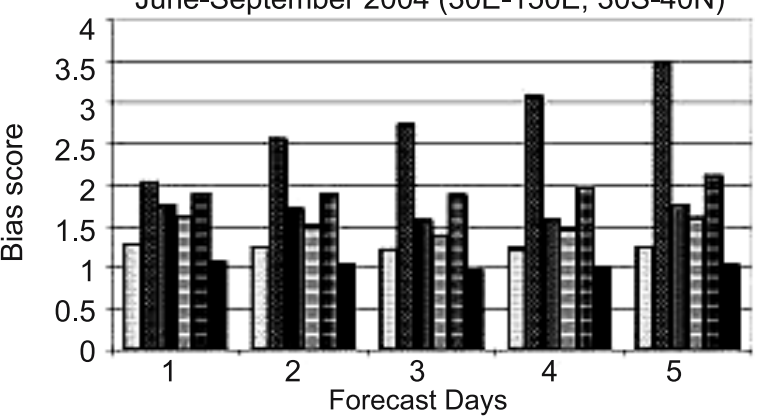

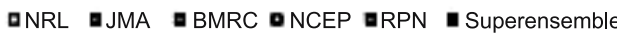
Precip Bias score (2 mm/day threshold)

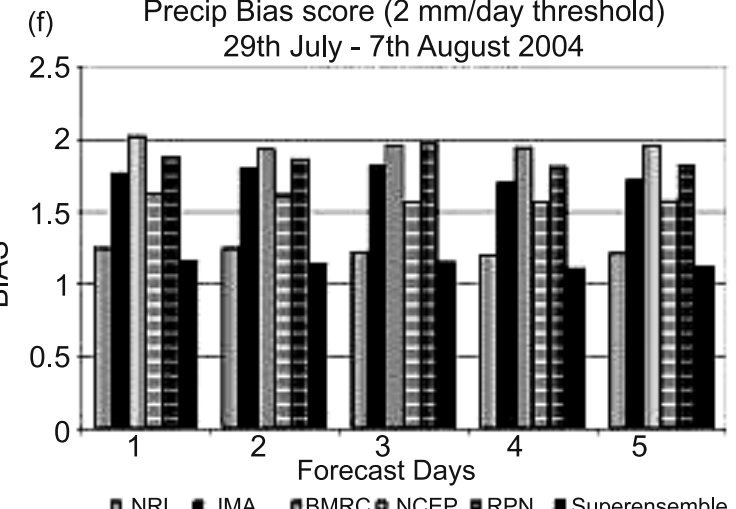

(h) Precip Bias score (5 $\mathrm{mm} /$ day threshold)

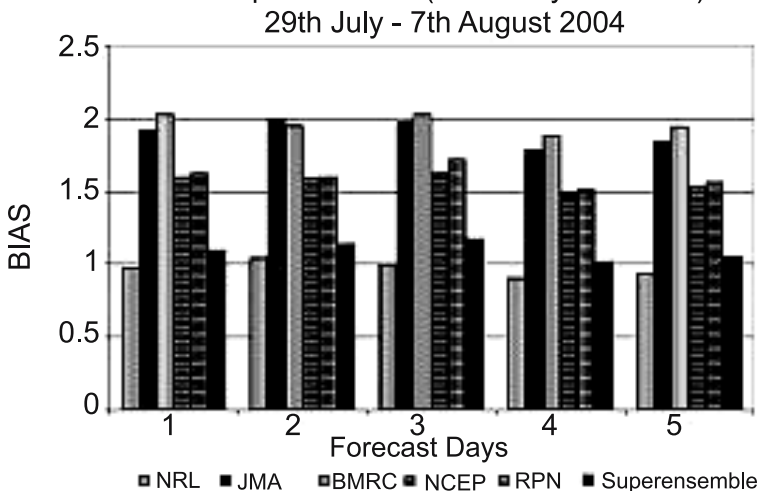

Figure 6. Forecast skills of multimodel superensemble precipitation compared against the member models.

forecasts. It has been possible to maintain a realtime website (http://lexxy.met.fsu.edu/rtnwp/) for global numerical weather prediction from the construction of a multimodel superensemble. These are the current best known global operational NWP models. It is possible to obtain the highest 
skills on a daily basis out to 6 days both globally and regionally from the superensemble. The fields we have looked at include the winds, temperature, mean sea level pressure, geopotential heights at $500 \mathrm{hPa}$, humidity, and precipitation. The skill matrices include RMS error, anomaly correlation, and probabilistic measure such as equitable threat scores and bias score for precipitation. We have noted some extraordinary improvements in skill over all models. The overlaying of the geopotential height isopleths over the globe for day 5 of forecasts and corresponding analysis (observation) display a line-for-line match. These carry anomaly correlation that exceeds 0.9 on day 5 . These improvements in global NWP are well beyond those that were possible a few years ago. We have also seen major improvements in the forecasts over the southern hemisphere where the skills of forecasts are close to those of northern hemisphere.

The monsoon forecasts by member models especially for monsoon rainfall were one of the difficult areas for the global NWP. Here the errors of the member models were quite large. The use of the multimodel superensemble reduced these errors considerably, and it became possible to obtain much higher equitable threat scores for the day 1 through day 5 forecasts over a monsoon domain. On day 5 of forecast, it became possible to consistently maintain anomaly correlations for sea level pressure over the monsoon region at values close to 0.92 . The member models best values were considerably lower. This illustrates the robust strength of the multimodel superensemble. Similar results were noted for the wind components at $850 \mathrm{hPa}$ and $200 \mathrm{hPa}$ levels.

The reduction of collective bias error by the superensemble (derived from the statistics of the training phase) makes it possible to reduce errors in the real time forecast phase. We have also examined the error of the ensemble mean for some variables. We noted that those were generally lower compared to those of the member models. However, when compared to the errors of the multimodel superensemble the errors of the ensemble mean were higher consistently. The ensemble mean assigns weights of $1 / N$ ( $N$ being the number of member models) uniformly over the globe for all models. The superensemble, in this regard, is very selective regionally, and assigns fractional, positive and even negative weights at different grid locations for the member models. In all we make use of as many as $10^{7}$ weights for 3-dimensional grid location $(384 \times 142 \times 10)$ for roughly 7 variables and 7 member models. The error correction plays a major role in providing the best global NWP product on real time. We also illustrate a case study of a monsoon depression where the improvements of forecast details from the superensemble over those of the member models are highlighted.

\section{Acknowledgements}

We gratefully acknowledge the ECMWF for providing analysis. We wish to acknowledge the member modeling groups whose forecast datasets were used in this paper. The research reported here was supported by NSF grant numbers ATM-0419618 and NAG5-13563. We are also thankful to our reviewers for their useful suggestions.

\section{Appendix 1}

\section{Equitable Threat Score}

The equitable threat score (Schaefer 1990) is defined as

$$
E T S=(H-C H) /(F+O-H-C H)
$$

where $F=$ the number of grid boxes that forecast more than the threshold, $O=$ the number of grid boxes that observe more than the threshold, $H=$ the number of grid boxes that correctly forecast more than the threshold, $\mathrm{CH}=$ the expected number of correct forecasts due to chance $=F \times$ $O / T$, where $T=$ the total number of grid boxes inside the verification domain.

The equitable threat score seems to be a good estimate for overall forecast skill. The higher the value, the better the forecast model skill for that particular threshold. The equitable threat score can vary from a small negative number to 1.0, where 1.0 represents a perfect forecast. This is basically the ratio of the correct forecast area to the total area of the forecast and observed precipitation. The model gets penalized for forecasting rain in the wrong place as well as not forecasting rain in the right place. Thus, the model with the highest score is generally the model with the best forecast skill.

\section{Bias score}

The bias score is a very simple equation, defined as simply as $F / O$. This score does not comment at all on the skill of a model forecast in terms of the placement of precipitation, but does give an indication if a model is consistently over- or under-forecasting areas of precipitation. The best model is generally the one that remains near the 1.0 line, which means that the model does not generally over-forecast precipitation or under-forecast precipitation. If the model verifies over 1.0, it is over-predicting precipitation, and if below 1.0, it is under-predicting precipitation. 


\section{List of Acronyms}

3D-VAR

4D-VAR

AGCM

AIRS

AMSU

BMRC

$\mathrm{CMC}$

CMORPH

DMSP

ECMWF

EMC
ERS
FNMOC

FSU

GASP

GEM

GFS

GOES

GOMOS

GSM

HIRS

ITCZ

JMA

MODIS

MSU

NASA

NCAR

NCEP

NCMRWF

NESDIS

NOAA

NOGAPS

NRL

QuikScat

RMS Error
Three Dimensional Variational

Data Assimilation

Four Dimensional Variational

Data Assimilation

Atmospheric General Circulation

Model

Advanced InfraRed Sounder

Advanced Microwave Sounding

Unit

Bureau of Meteorology Research

Centre, Australia

Canadian Meteorological Centre

CPC MORPHing technique

Defense Meteorological Satellite

Program

European Centre for

Medium-Range Weather Forecasts

Environmental Modeling Center

European Remote Sensing

Fleet Numerical Meteorology and

Oceanography Center

Florida State University

Global AnalysiS and Prediction

Global Environmental Multiscale

Model

Global Forecast System

Geostationary Operational

Environmental Satellites

Global Ozone Monitoring by

Occultation of Stars

Global Spectral Model

High Resolution Infrared

Radiation Sounder

Inter Tropical Convergence Zone

Japan Meteorological Agency

Moderate Resolution Imaging

Spectroradiometer

Microwave Sounding Unit

National Aeronautics and Space

Administration

National Center for Atmospheric

Research

National Center for

Environmental Prediction

National Centre for Medium

Range Weather Forecasting

National Environmental Satellite,

Data, and Information Service

National Oceanic \& Atmospheric

Administration

Navy Operational Global

Atmospheric Prediction System

Naval Research Laboratory

Quick Scatterometer

Root Mean Square Error
RPN Recherche Prévision Numérique

SBUV Solar Backscatter Ultraviolet radiometer

SLP Sea Level Pressure

SSM/I Special Sensor Microwave

Instrument

SST Sea Surface Temperature

SVD Singular Value Decomposition

SYNOP Traditional Synoptic Weather

Observations

TRMM Tropical Rainfall Measuring

Mission

UKMet United Kingdom Met. Office

\section{References}

Arakawa A and Schubert W H 1974 Interaction of a cumulus cloud ensemble with the large-scale environment. Part I; J. Atmos. Sci. 31 674-701.

Businger J A, Wyngard J C, Izumi Y and Bradley E F 1971 Flux profile relationship in the atmospheric surface layer; J. Atmos. Sci. 28 181-189.

Charnock H 1955 Wind stress over a water surface; Quart. J. Roy. Meteor. Soc. 81 639-640.

Edwards J M and Slingo A 1996 Studies with a flexible new radiation code I: choosing a configuration for a large-scale model; Quart. J. Roy. Meteor. Soc. 122 689-719.

Ferraro R R and Marks G F 1995 The development of SSM/I rain-rate retrieval algorithms using ground-based radar measurements; J. Atmos. Oceanic Technol. 12 755-770.

Grell G A 1993 Prognostic evaluation of assumptions used by cumulus parameterizations; Mon. Wea. Rev. 121 764-787.

Harshvardan and Corsetti T G 1984 Longwave parameterization of the UCLA/GLAS GCM; NASA Technical Memorandum 86072 , Goddard Space Flight Center, Greenbelt, MD, 52pp.

Harshvardhan, David A Randall and Thomas G Corsetti 1989 Earth Radiation Budget and Cloudiness Simulations with a General Circulation Model; J. Atmos. Sci. 46 1922-1942.

Joyce R J, Janowiak J E, Arkin P A and Xie P 2004 CMORPH: A method that produces global precipitation estimates from passive microwave and infrared data at high spatial and temporal resolution; J. Hydromet. $\mathbf{5}$ 487-503.

Krishnamurti T N 1979 Tropical meteorology. WMO Publ. No. 364, Compendium of Meteorology, Part 4, 428pp.

Krishnamurti T N, Low-Nam S and Pasch R 1983 Cumulus parameterization and rainfall rates II; Mon. Wea. Rev. $111816-828$.

Krishnamurti $\mathrm{T}$ N, Xue J, Bedi H S, Ingles $\mathrm{K}$ and Oosterhof D 1991 Physical initialization for numerical weather prediction over the tropics; Tellus 43 53-81.

Krishnamurti T N, Bedi H S and Ingles K 1993 Physical initialization using the SSM/I rain rates; Tellus $\mathbf{4 5 A}$ 247-269.

Krishnamurti $\mathrm{T}$ N, Kishtawal C M, LaRow $\mathrm{T}$ E, Bachiochi D R, Zhang Z, Williford C E, Gadgil S, Surendran S 1999 Improved weather and daily and medium Range climate forecasts from multi-model superensemble; Science 285 1548-1550.

Krishnamurti T N, Kishtawal C M, Shin D W and Williford C E 2000a Multimodel ensemble forecasts for weather and seasonal climate; J Climate 13 4196-4216. 
Krishnamurti T N, Kishtawal C M, Shin D W and Williford C E 2000b Improving tropical precipitation forecasts from a Multianalysis Superensemble; J Climate 13 4217-4227.

Krishnamurti T N, Surendran S, Shin D W, CorreaTorres R J, Vijay Kumar T S V, Williford E, Kummerow C, Adler R F, Simpson J, Kakar R, Olson W S and Turk F J 2001 Real-time multianalysismultimodel superensemble forecasts of precipitation using TRMM and SSM/I products; Mon. Wea. Rev. 129 $2861-2883$.

Krishnamurti T N, Rajendran K, Vijaykumar T S V, Stephen Lord, Zoltan Toth, Xiaolei Zou, Steven Cocke, Jon E Ahlquist and Michael Navon I 2003 Improved Skill for the Anomaly Correlation of Geopotential Heights at $500 \mathrm{hPa}$, Mon. Wea. Rev. 131 1082-1102.

Kuo H L 1974 Further studies of the parameterization of the influence of cumulus convection on large-scale flow; J. Atmos. Sci. 31 1232-1240.

Lacis A A and Hansen J E 1974 A parameterization for the absorption of solar radiation in the Earth's atmosphere; J. Atmos. Sci. 31 118-133.

Louis J F 1979 A parametric model of vertical eddy fluxes in the atmosphere; Bound.-Layer Meteor. 17 $187-202$

Louis J F, Tiedtke M and Geleyn J F 1982 A short history of the operational PBL parameterization at ECMWF; ECMWF Workshop on Planetary Boundary Parameterizations, 59-79.

Moorthi S and Suarez M J 1992 Relaxed Arakawa-Schubert: a parameterization of moist convection for general circulation models; Mon. Wea. Rev. 120 978-1002.
Palmer T N, Shutts G J and Swinbank R 1986 Alleviation of a systematic westerly bias in general circulation and numerical weather prediction models through an orographic gravity wave drag parameterization; Quart. J. Roy. Meteor. Soc. 112 1001-1039.

Pan Hua-lu and Wan-Shu Wu 1994 Implementing a mass flux convective parameterization package for the NCEP Medium-range forecast model. Preprints of the 10th AMS Conf. on Num. Weather Pred., July 18-22, 1994, Portland OR (Pan H-L and Wu W-S 1995 NMC Office Note, No. 409, 40pp.)

Rao Y P 1976 South West Monsoon IMD Publications.

Schaefer J T 1990 The critical success index as an indicator of warning skill; Wea. Forecasting 5 570-575.

Schwarzkopf M D and Fels S B 1991 The simplified exchange method revisited: An accurate, rapid method for computation of infrared cooling rates and fluxes; J. Geophys. Res. 96 9075-9096.

Troen I and Mahrt L 1986 A simple model of the atmospheric boundary layer: Sensitivity to surface evaporation; Bound.-Layer Meteorol. 37 129-148.

Wilks D S 1995 Statistical Methods in the Atmospheric Sciences, Academic Press, 467pp.

Yanai M, Esbensen S and Chu J 1973 Determination of bulk properties of tropical cloud clusters from large-scale heat and moisture budgets; J. Atmos. Sci. 30 611-627.

Yun W T, Stefanova L, Mitra A K, Vijay Kumar T S V, Dewar W and Krishnamurti T N 2005 Multi-Model Synthetic Superensemble Algorithm for Seasonal Climate Prediction using DEMETER Forecasts; Tellus $\mathbf{5 7}$ $280-289$. 